\title{
Identifying Optimum Taper Lengths for Zipper Merging Applications using Real Data and Microscopic Simulation
}

\author{
Wakeel Idewu' ${ }^{1}$ Pattanun Chanpiwat ${ }^{2}$, Hana Naghawi ${ }^{3 *}$ \\ ${ }^{1}$ Department of Civil and Environmental Engineering, Virginia Military Institute, 610 Nichols Hall, Lexington, VA, 24450, USA \\ 2 Department of Mechanical Engineering, University of Maryland, College Park, MD, 20742, USA \\ ${ }^{3}$ Civil Engineering Department, School of Engineering, The University of Jordan, Al-Jubeiha Street, Amman, Jordan \\ * Corresponding author, e-mail: h.naghawi@ju.edu.jo
}

Received: 28 August 2018, Accepted: 19 November 2019, Published online: 14 June 2019

\begin{abstract}
Motorists lack of understanding on the proper way to maneuver through lane closures during congested periods cause driver confusion. This confusion directly and indirectly creates inconsistent flow patterns, forced merges, travel time delays, and crashes. Engineers and developers have tried to improve the merge systems used in construction zones to reduce driver frustration, improve travel time, and increase safety. Encouraging drivers to use the zipper merge approach has been assumed by some to target these issues. When implemented, drivers jointly merge together in an alternating fashion at two-to-one lane closures/reductions. There is a difference in opinion between traffic officials concerning the taper length required to efficiently accommodate these types of merging patterns - particularly those that occur near construction sites. Current practice uses the taper design guideline presented in the MUTCD. However, some believe this unique approach to merging at lane reductions should be accompanied by a shorter/longer taper. This study simulated 192 scenarios consisting of eight different percent truck compositions, six different transition lengths, and four different traffic volumes in VISSIM. The simulation models were calibrated with field data taken while a zipper merge configuration was in operation on a freeway. The main objective was to identify the optimum transition length when placing a zipper merge configuration because it visually and physically promoted alternating merging maneuvers. The results indicated none of the six tested taper lengths had a clear advantage over the other under multiple traffic volumes and truck percentages. Although statistically equal, operational differences in response to taper lengths were present and became more pronounced as volumes and truck percentages increased.
\end{abstract}

Keywords

zipper merge, alternating merge, joint merge, taper length, truck percentages, lane reduction, merging operations, work zone

\section{Introduction}

Lane reductions are a common feature in the United States and are structured as shown in Fig. 1. A key feature to this configuration is the taper length, which is the length required to effectively reduce the number of lanes on a roadway. It is common and practical to reduce lanes in areas where less capacity is required and after the termination of auxiliary/passing-relief lanes on rural 2-lane roads. Perhaps the most common are the lane reductions observed just before a construction zone were a lane is closed for construction. Tapers accompany lane closures. They restrict the longitudinal movement near the merge point and are used to channel vehicles from the terminating lane into an adjacent continuing lane. The procedure for determining the length required for each taper is detailed in the Manual of Uniform Traffic Control Devices (MUTCD). General design practice makes use of the following equations:

For speeds greater than $45 \mathrm{mph}$

$L=W \times S$

And for speeds less than $45 \mathrm{mph}$

$L=\frac{W \times S^{2}}{60}$

Where:

$W=$ the width of the lane (feet)

$S=$ the posted speed, $85^{\text {th }}$ percentile speed, or the calculated speed (mph), and

$L=$ the longitudinal (taper) length of the transition zone (feet) 


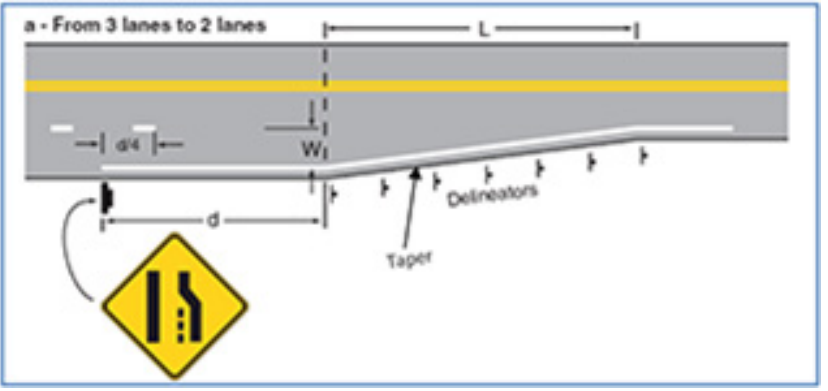

Fig. 1 Lane reduction and taper layout

This equation accounts for the three to four seconds drivers have been observed to need when merging into an adjacent lane. Therefore, the length a vehicle will travel while merging is dependent on the vehicle's speed and the lateral distance required to complete the merge.

Most lane reductions result in vehicles in the closed lane merging with vehicles in an adjacent open lane. Familiar drivers usually position themselves in the open lane before the taper. Drivers in the closed lane must yield to those in the open lane to which right of way (ROW) is typically assigned.

By use of a taper, the current lane reduction design specified in the MUTCD seeks to guide drivers from an upcoming closed lane to an adjacent open lane. Such guidance can be unsafe and inefficient during congested periods. Since right lanes carry the most traffic and consequently the most load, they are closed for repairs more often than the left lanes. Drivers traveling in the slower moving right lane must merge with faster moving traffic in the left lane. During congested periods, however, drivers traveling in the dense and slower open lanes are at a disadvantage to the more aggressive drivers traveling in the faster-moving closed lane. This commonly leads to several safety related problems. An influx of traffic weakens merging operations causing motorists to tolerate and accept unsafe merging gaps near the merge point. Motorists traveling in the open lane(s) are less willing to cooperate with the motorists in the closed lane when long queues begin to form. Cases such as these are problematic and disrupt the intended purpose of merging areas.

In attempt to address the issues stated above, a popular strategy, more commonly used in countries such as the Netherlands and Germany, is being encouraged in the United States. During congested periods in Germany, motorists are required to follow a "zipper rule" in which drivers in the continuing lane permit adjacent vehicles to merge in an alternating pattern (see Fig. 2). In this instance, right-of-way assignment is suspended until the congested period ends, and as a result, less upstream lane changing

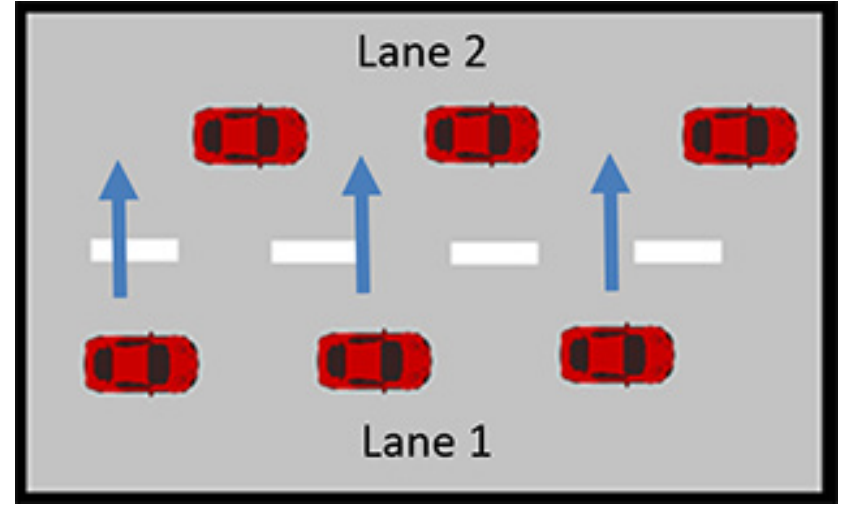

Fig. 2 Zipper merge arrangement between two lanes

occurs. Lessening the advantage of changing lanes also decreases the potential for side-swipe crashes. Additionally, less weaving between lanes improves traffic operations.

In the United States, the Minnesota Department of Transportation, promotes the zipper merge strategy by advertising and demonstrating it on its website. The zipper merge strategy has been suggested to operate more efficiently during peak periods, and safely maintain the "merging fairness" many drivers desire. However, some critics of the strategy contend that it is not practical since many drivers are inherently selfish and both lanes must cooperate even though one lane appears to have priority over the other.

\section{Literature review}

While popular in Europe, the zipper merge is less practiced in the United States. The first live attempt was made on a mock construction site placed near a rural freeway in 2008. By physically creating a two-sided taper near the merge point, researchers compared traffic operations using a zipper merge to traffic operations using the more traditional MUTCD taper configuration. The zipper manuever was prompted by channeling devices placed on both sides of the right and left lanes to create a two sided taper and an alternating merging pattern in the middle of a roadway. Usint this method traffic can be shifted back to either the left or right lane opposite of construction, as shown in Fig. 3.

The zipper merge is thought to improve the capacity of the merge area, to cause a smoother flow of traffic, and to reduce lane changes prior to reaching the merge area. It also satisfies drivers in both lanes since drivers in the closed lane do not have to compete to get into the open lane (Idewu and Wolshon, 2010a).

To date, the merging plan shown in Fig. 3 has been tested virtually, in the field during live construction, and in the field without construction (Aghazadeh and Ikuma, 2013; 


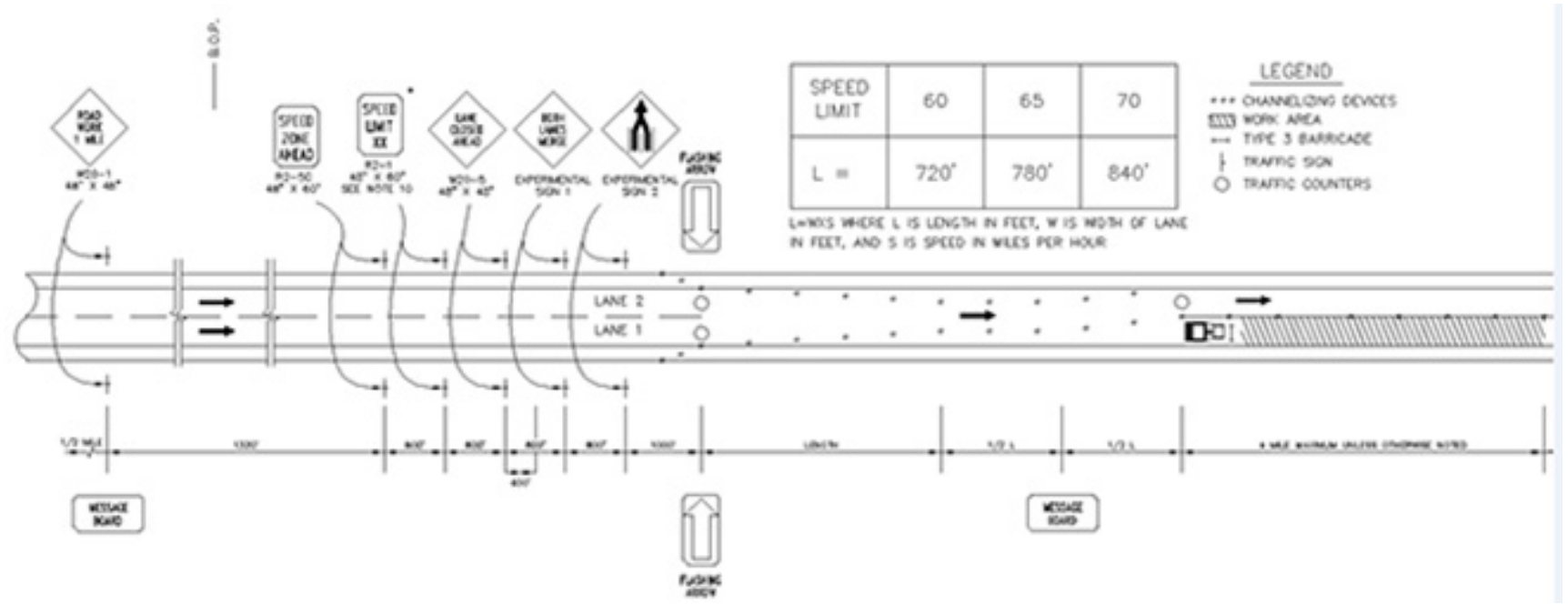

Fig. 3 Zipper merge traffic control design and plan in construction zone (Idewu and Wolshon, 2010a)

Rayaprolu et al., 2010; Costa, 2013; Idewu and Wolshon, 2010). Results were similar for each test. In 2012, the Canadian Ministry of Transportation implemented the zipper merge configuration for a portion of the Highway 401 and Highway 40 Interchange Reconfiguration project. It was erected for 13 days at least 12 hours per day. Zero crashes were reported while it was implemented in the field. Similar to the virtual test and live field tests, volume between lanes were balanced, speeds were slower at the taper, but traffic flow through the site was unaffected by the zipper merge configuration. Most motorist responded as intended to the two-sided taper in an alternating pattern.

Drivers intuitively understand the alternating merging concept and seem to be accepting of the method. There is also less braking and lane changing between lanes before the taper (Idewu and Wolshon, 2010b). The greatest and most inconsistent difference between the zipper merge and the more traditional merge method is merging distance. The distance at which vehicles begin to merge varies by volume and vehicle type when the zipper merge is used.

During low volume periods, drivers are more likely to take turns merging at the defined merge point, as intended. As volumes approach capacity, the location where merging begins moves further upstream. The exact merging location varies and is governed by the leading vehicle in the arriving platoon and their distance from the taper. If the leading vehicle arrives at the taper of the zipper merge in the middle lane, the following vehicles will begin to merge into the center of the roadway. Similarly, if the leading vehicle reaches the taper in either the left or right lane, the following vehicles in the platoon will mimic the leading vehicle.
Most truck drivers tend to merge and stay in the middle of the road (over the dashed lines) earlier than the passenger cars. These occurrences were captured by video which was observed by the researchers, and observed during a past driving simulation study (Southwest Region Transportation Center, 2013; Idewu, 2009).

According to MUTCD, the merging taper length on a 70 mile per hour road having a width of 12 feet should be 840 feet. It is also required that the length of any shifting taper be half the length of the merging taper.

While the zipper merge practice is documented as having several advantages, there is one obvious constructability disadvantage. The number of channeling devices required for the zipper merge at construction sites greatly exceeds that required for the traditional MUTCD merge. The total length of the first, second, and third combined segments of the zipper merging area is 1,680 feet [assuming a 70 mile per hour road]. Thus, the zipper merge configuration is twice as long and will require more than twice as many channeling devices to set up its taper.

Whether a zipper merge or simply and area where alternating merging is required, the most critical location, shown in Fig. 1, is the area where two lanes are transitioning to one. The most critical aspect of that area is the taper length. The next section shares two opinions of the taper length and describes the process of setting the twosided tapers in the field.

\section{Zipper merge taper lengths and uses}

A clear construction advantage of the zipper merge configurations is that light work, such as shoulder/abutment maintenance, can be performed on both sides of the roads 
simultaneously. For projects that require work on both sides, switching a lane closure from one side to the other is relatively simple and efficient. One would only need to change the direction of the third segment as shown in Fig. 3. This decreases the tear down and set up time of traffic control devices at the same location.

There is varying opinion on the length of taper that best suits the zipper/alternating merge strategy. Taper length preference seems to vary by job function. Maintenance crews and contractors prefer a longer taper assuming the gradual transition would enhance worker safety. From a construction manager's perspective, a more gradual transition would decrease crash potential and the complexity associated with merging (Macaluso, 2008).

Conversely, some scholars have recommended that shorter taper lengths be used for the zipper/alternating merge application. Tapers that are too long could give the false perception that merging can occur well after entering into the transition zone where the capacity is steadily decreasing, thereby negatively effecting traffic performance. While both arguments are sound, neither can be confirmed since few studies on optimum taper lengths for alternating merge applications are performed. This study seeks to improve the understanding of taper lengths by evaluating the effects of varied taper lengths using the zipper merge configuration.

\subsection{Field set up of Two-Sided Taper}

The most efficient and safe approach to setting a two-sided taper is detailed below. These steps are the results of practices and observations made from live field tests. Proper signage, personnel, and equipment were obtained before beginning this process.

First, all required static signs were placed in their respective locations (Fig. 4a). This includes the Changeable Message Boards and one of the two Flashing Arrow Boards shown in Fig. 3.

Second, channeling devices were set one lane at a time. It's preferable to start on the side of the lane that will ultimately be closed. For instance, if the right lane will be closed, align the right side with channeling devices first (Fig. 4b).

Next, channeling devices along the continuous lane were set. This would be the left side, if the right lane is closed, and the right side if the left lane is closed (Fig. 4c). By this time, most vehicles begin to merge towards the center of the road before reaching the taper.

Finally, ensure the proper reflective features and beacon lights on channeling devices are functioning per the respective managing authorities' guidelines (Fig. 4d). Because this arrangement was placed on a high-speed freeway, the channeling devices were kept a lateral distance equal to at least sixteen feet. It is important to note the configurations were set up during low volume periods to limit any confusion between motorists approaching the tapers from both lanes.

Other options include:

1. Using pace vehicles to provide a level of protection when setting channeling devices; and

2. Employing the services of patrol officers. Simply having a patrol car present would decrease speeds and increase compliance while setting the second set of channeling devices.

\section{Scope}

Once the two-sided taper was set, traffic operation data were gathered to evaluate the efficiency and response to an alternating merge strategy near a construction site. As mentioned earlier, the distance at which drivers begin to alternate merges is dependent on several factors including: volume, truck percentages, and positioning of the leading vehicle. The degree to which the length of taper affects each of these factors has yet to be determined. The goal of this research was to identify the ideal taper length(s) that are best suited for cars and trucks approaching an alternating merge lane closure under various truck percent compositions and traffic volumes. The following section discusses the methodology used in simulating various merging scenarios to help identify taper lengths that are most appropriate for arriving traffic streams. The research reported here used a calibrated model to produce 192 scenarios by evaluating six different transition lengths, eight different truck percentages, and four different traffic volumes.

\section{Data collection for calibration}

The field data used to calibrate the zipper merge model for this research was collected from a rural freeway for approximately two weeks while the zipper merge configuration was in use. Magnetic vehicle imaging (MIR) recorders were affixed to the pavement surface at several locations before the taper, in the work zone's advance warning area. One MIR was placed after the taper. Speed and volume data were recorded in each lane. Those data were used to calibrate and validate the traffic simulation model at the microscopic level. VISSIM was the microscopic simulation package of choice since the zipper configuration has a unique geometric layout and required evaluation under multiple scenarios. 


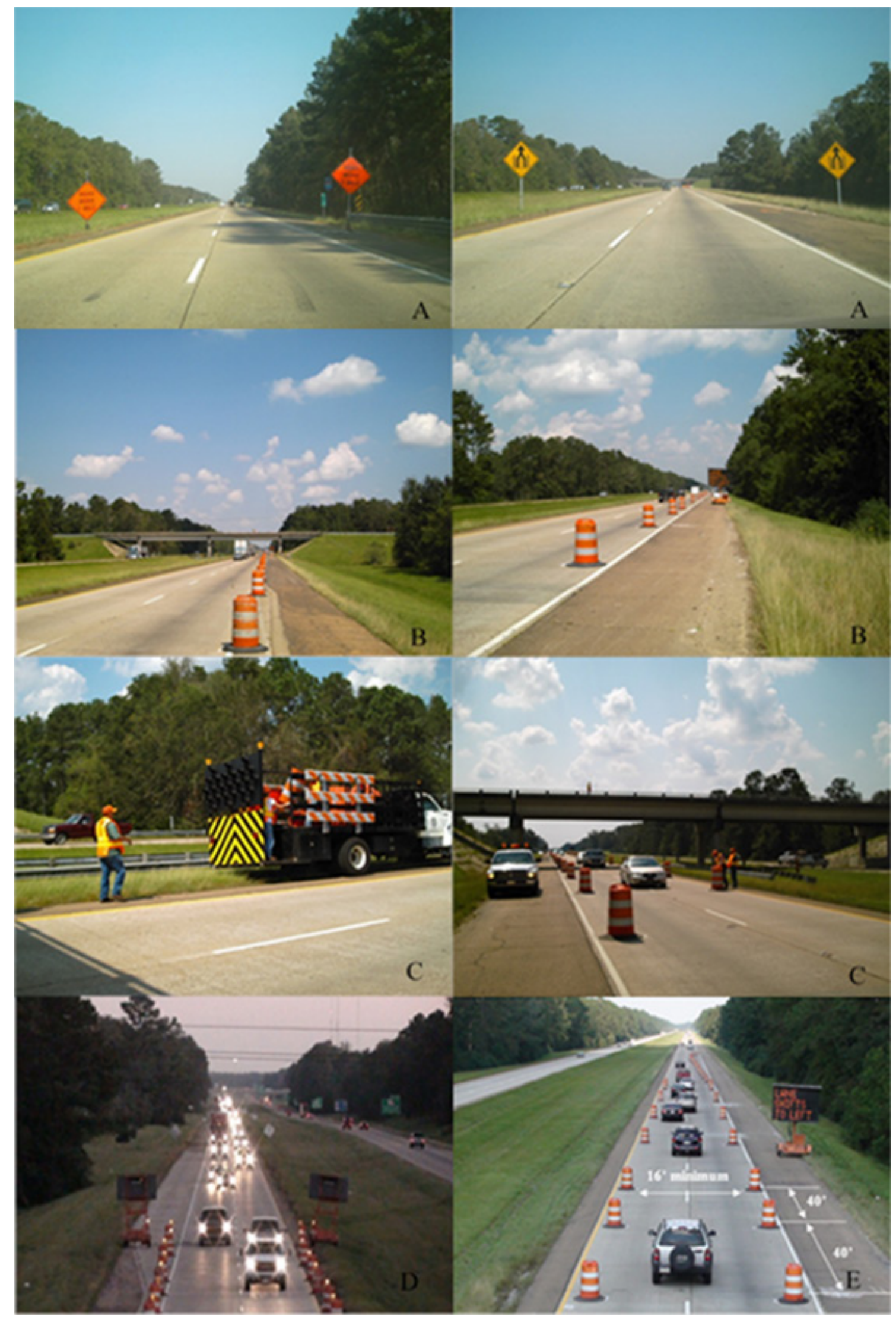

Fig. 4 Set up Progression of a Two-Sided Taper (a) static signs (b) closed lane tapers (c) open lane-opposite side tapers (d) beacon lights (e) channel device spacing 
The zipper merge operation was evaluated using eight different truck percentages, six different transition lengths and four different traffic volumes. Table 1 shows the variations that were evaluated, 192 in all.

\section{Percent truck composition}

This research varied the percent of trucks from zero to sixty percent at five, ten and fifteen percent increments. Some roads carry high truck traffic with percentages reaching 58.31\% (State of California, 2008). Others have much lower truck presence. Although 60 percent truck traffic has been observed in the past, those observation are less frequent than the lower and more common truck percentages values that range from 5 to 30 percent (Statistics, 2009). The truck percentages used in this study were selected to capture the affects taper lengths have on truck percentages, both common and uncommon.

Video observations of truck drivers showed that many of them merge early, regardless of the merging system used. Coupled with the finding that the average speed of trucks on interstates is four to seven miles per hour below that of passenger cars (Indrajit Chatterjee, 2009; Rayaprolu et al., 2013), the potential for trucks to drastically affect traffic flow at work zones is high.

Modifying taper lengths to match the truck demands may discourage the need to use the potentially dangerous tactics practiced by truck drivers. A distinctive tactic used by some is referred to as lane straddling. This is done by one truck straddling two lanes in effort to control and decrease the number of "late mergers." Another unique behavior of truck drivers is the formation of "rolling blockades." This happens when two trucks drive side by side preventing vehicles from passing them (McCoy, 2001). In doing so, the blockades could encourage more dangerous maneuvers such as motorists driving on shoulders and adding to driver frustration.

Table 1 Evaluated truck, transition length, and volumes interactions

\begin{tabular}{cccc}
\hline$\#$ & $\begin{array}{c}\text { \% Truck } \\
\text { Composition }\end{array}$ & $\begin{array}{c}\text { \% of Transition Length } \\
(\mathrm{ft}) \text {-Level }\end{array}$ & $\begin{array}{c}\text { Traffic Volume } \\
\text { (vph)—Level }\end{array}$ \\
\hline 1 & 0 & L1: $55 \%(462 \mathrm{ft})$ & 1,000 \\
2 & 5 & L2: $70 \%(588 \mathrm{ft})$ & 1,500 \\
3 & 10 & L3: $85 \%(714 \mathrm{ft})$ & 2,000 \\
4 & 15 & L4: $100 \%(840 \mathrm{ft})$ & 2,500 \\
5 & 20 & L5: $115 \%(966 \mathrm{ft})$ & \\
6 & 30 & L6: $130 \%(1092 \mathrm{ft})$ & \\
7 & 45 & & \\
8 & 60 & & \\
\hline
\end{tabular}

\section{Taper lengths}

As stated earlier, some believe shorter transition lengths would increase the traffic performance, but others state the opposite. A two-to-one lane reduction on a $70 \mathrm{mph}$ road would typically use an 840 foot taper, if guidelines in the MUTCD are followed. This length may or may not be suitable for the zipper merge configuration. The research reported in this paper conduct experiments on six different taper lengths at $55 \%$ (462 ft); $70 \%$ (588 ft); 85\% (714 ft); $100 \%$ (840 ft); $115 \%$ (966 ft); and $130 \%$ (1092 ft) of the standard transition length 840 feet.

\section{Traffic volume}

The zipper merge configuration was tested using several input volumes starting at 1,000 vehicles per hour (vph) and increasing to $2,500 \mathrm{vph}$ at $500 \mathrm{vph}$ increments. Past studies have shown that the capacity for 2-tol lane closures ranges from 1,500 vph to 1,600 vph (Dixon et al., 1996; Jiang, 1999; Maze et al., 2000; Racha et al., 2008). Weekday traffic from $4 \mathrm{pm}$ to $6 \mathrm{pm}$ were used to calibrate the model in VISSIM. The average traffic volume and percent truck composition of the six weekdays used in this study were approximately 1,447 vehicle/hour (vph) and 11 percent truck composition. The highest traffic volume exceeded capacity and the highest truck percentage was recorded at 13 percent. The four volumes selected for testing (after calibration) are as follows: $1,000 \mathrm{vph} 1,500 \mathrm{vph}, 2,000 \mathrm{vph}$, and 2,500 vph. Each input volume, was analyzed against several truck percentages and taper lengths resulting 192 study scenarios.

\section{Coding and calibration of simulation models}

Prior to constructing the model, live lane specific volume and speed data was captured at the zipper merge site before the taper and at one location immediately following the taper. The network constructed in VISSIM consisted of the Entrance Area, Advance Warning Area, Transition Area, Buffer Zone, Work Zone, Termination Area, and Exit as shown in Fig. 5.

Six of the highest weekday traffic between 4:00 p.m. and 6:00 p.m. was selected from the field data to calibrate the model. These two-hour periods were parsed into fifteen-minute interval periods. Each interval had an associated traffic volume, class (passenger car or truck), and speed. The average speed, volume, and percent truck composition of those intervals were used as speed, volume, and percent truck inputs for the simulation.

The lane specific field data captured at different locations within the advance warning areas revealed that 
speeds dropped as motorists neared the taper. This phenomenon was replicated in VISSIM by setting the desired speed decision point near the "Speed Limit" signs, 3,400 feet before the $1^{\text {st }}$ zipper merge area segment. Data collection points were set to collect speed and volume on both lanes at 6,120 feet, 3,040 feet, and 840 feet before the taper, in additions to, at the taper, and 840 feet after the taper. These locations closely matched the locations where field data was collected.

The default parameters in Wiedemann 99 driving behavior car following and lane change models were modified for calibration. Ten different parameters, $\mathrm{CC} 0-\mathrm{CC} 9$, were available for adjustments. These parameters had a direct impact on the vehicle interaction in and between lanes for freeway operation. Chitturi and Benekohal (2008) stated simulated capacity values for freeways were influenced by standstill distance ( $\mathrm{CC} 0$ ) and headway time (CC1) (Chatterjee, 2009), where $\mathrm{CC} 0$ was the preferred distance in feet between stopped vehicles with no variation (PTV, 2009), and CC1 is defined as the time in seconds that a driver wants to keep between his vehicle and the next in order to maintain a safe driving distance. It was found that increasing the $\mathrm{CCl}$ value resulted in more cautious driving. Since drivers keep relatively safe distances while following other vehicles, the safety distance became the value that had the strongest influence on capacity (PTV, 2009). Other peripheral parameters such as minimum headway (front/rear) recommended by Yang et al.(2009) as well as CC1 (Headway Time), CC2 (Following Variation), and Safety distance reduction factor (SRF factor), recommended by Indrajit et al. (2009), were also explored during the calibration process.

This research performed the calibrations by adjusting each parameter at one-tenth increments while fixing the rest of the parameters. This procedure was carried out for each parameter from $\mathrm{CC} 0$ to $\mathrm{CC} 9$ (i.e. standstill distance, headway time, following variation, following deceleration, negative and positive speed differentials, speed oscillation, oscillation acceleration, standstill acceleration, and acceleration at $80 \mathrm{~km} / \mathrm{h}$ ) until the throughput volumes at the end of the zipper merge and lane specific speeds at each counter location were consistent with the field data collected. Ten simulations with different random seeds were run for each change. The simulation outputs and field data were statistically compared at the 95 percent confidence level. The calibrated models were validated in a similar fashion, but instead, lane specific volume at several counter locations, 3040 feet and 840 feet before

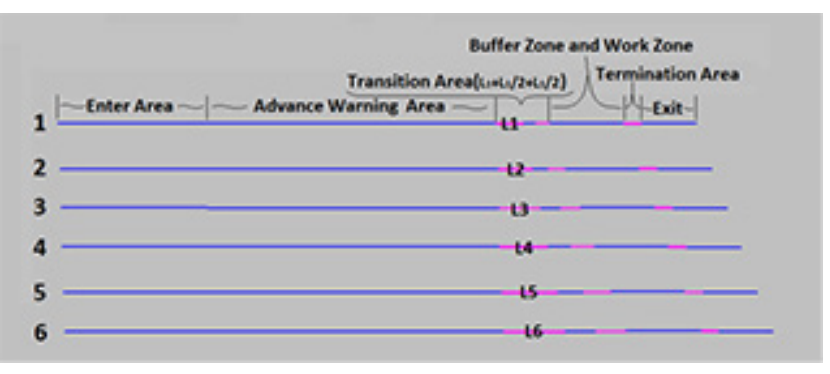

Fig. 5 A set of zipper merge configuration with six different coded transition lengths (L1-L6)

the taper, as well as 840 feet after the taper were statistically compared with the relative field data. As a result, the default parameter for $\mathrm{CC} 0$ was changed to 4.99 feet and the other default values for the car following and lane change parameters remained unchanged, as they had no substantial impact to driving behavior for a configuration like the zipper merge.

\section{Simulation tests}

The simulation data was collected on eight-replica sets of roads for each truck percentage $(0 \%, 5 \%, 10 \%, 15 \%$, $20 \%, 30 \%, 45 \%$, and $60 \%$ ). Each set consisted of six roads that varied on transition length and input volume. Boundaries spanning from the Advance Warning Area to the end of the $3^{\text {rd }}$ zipper merge transition segment were analyzed using the polygon node function. Measures used in the evaluation were vehicle throughput volume and average number-of-stops. The throughput volume and number of stops that occurred within the entire study area (from the beginning of the advance warning area through the transition zone) was analyzed at fifteen-minute intervals. Simulation analysis began after a warm-up period of 900 seconds, which loaded vehicles onto the network. An hour worth of data were collected for each of the 192 scenarios. The scenarios were run ten times each by the multirun function in VISSIM with ten different random seeds and then averaged.

\section{Analysis of simulation results}

This study compared vehicle throughput and average number of stops per vehicle for each created scenario. These variables were thought to capture potential flow and safety impacts various taper lengths have on the zipper merge. A high number of stops are indicative of possible conflicts that can exist between two vehicles. Low throughput volumes are representative of traffic flow disturbances. The ideal taper length would be one that can 
safely accommodate high traffic volumes of all vehicle types. Therefore, the optimum taper length would produce high throughput volumes with very few stops.

The following results in this paper are sectioned by volume; $1000 \mathrm{vph}, 1500 \mathrm{vph}, 2000 \mathrm{vph}$, and $2500 \mathrm{vph}$. Each one of the four volume inputs were tested using six different taper lengths (L1-L6) and eight different truck percentages shown in Table 1. An analysis of variance (ANOVA) test was performed at 95 percent confidence level to determine if the output for each taper length statistically differed from the outputs of all other taper lengths. This was done for the four different input volumes. In the event a statistical difference was found, a comparison of means test was performed to identify which taper length(s) differed from the tested group.

\subsection{Low traffic volume $(1,000 \mathrm{vph})$}

An input volume of 1,000 vph on a 2-tol lane closure is considered low because it is approximately 38 percent below the capacity of one lane within a work zone. As mentioned earlier, the 2-to-1 lane closure capacity range is in-between 1,500 and 1,600 vehicles per hour. The low traffic volume is representative of general traffic conditions during non-peak hours.

Fig. 6 shows the affect trucks had on traffic operations for the zipper merge configuration. As anticipated, vehicles were not affected by truck presences or taper length since conflicts between vehicles were relatively low. The ANOVA test revealed that increasing/decreasing transition lengths had no significant effect on throughput volume or number-of-stops.

\subsection{Moderate traffic volume (1,500 vph)}

Statistical tests indicated that all transition lengths, L1-L6, produced equal results for throughput volume and numberof-stops. Unlike what was found for low traffic volumes, truck presence did slightly affect traffic operations. Fig. 7 shows the throughput volume slightly decreasing (about $4 \%$ ) when truck percentages reach 60 percent. Similarly, vehicle stops were almost nonexistent until truck percentages increased to 60 percent. Overall, the data suggests volumes equivalent to $1,500 \mathrm{vph}$ were found not to be impacted by truck presence or taper length, provided the truck presence was equal to or below 45 percent.

\subsection{High traffic volumes (Greater than 2,000 vph)}

Volumes greater than $1,500 \mathrm{vph}$ are either near or beyond the capacity of 2-to-1 lane closures. Therefore input volumes of $2,000 \mathrm{vph}$ or more will likely be affected by changes in traffic composition. Traffic flow was negatively correlated to truck percentage during periods of high traffic volumes. Trends seen in Fig. 8 show throughput volume decreasing as truck percentages increase. The highest throughput, 1,972 vph, was observed when zero trucks were present, whereas only 1,432 vph throughput volume was observed with a 60 percent truck volume. This suggests that throughput volumes can decrease as much as 27 percent when there is a high percentage of truck traffic on a road that is oversaturated with traffic. The number of stops was also observed to increase 27 percent as well, suggesting that a higher potential for crashes exist when there is a high truck presence within the peak hour. While zipper merge operation was affected by high volumes and

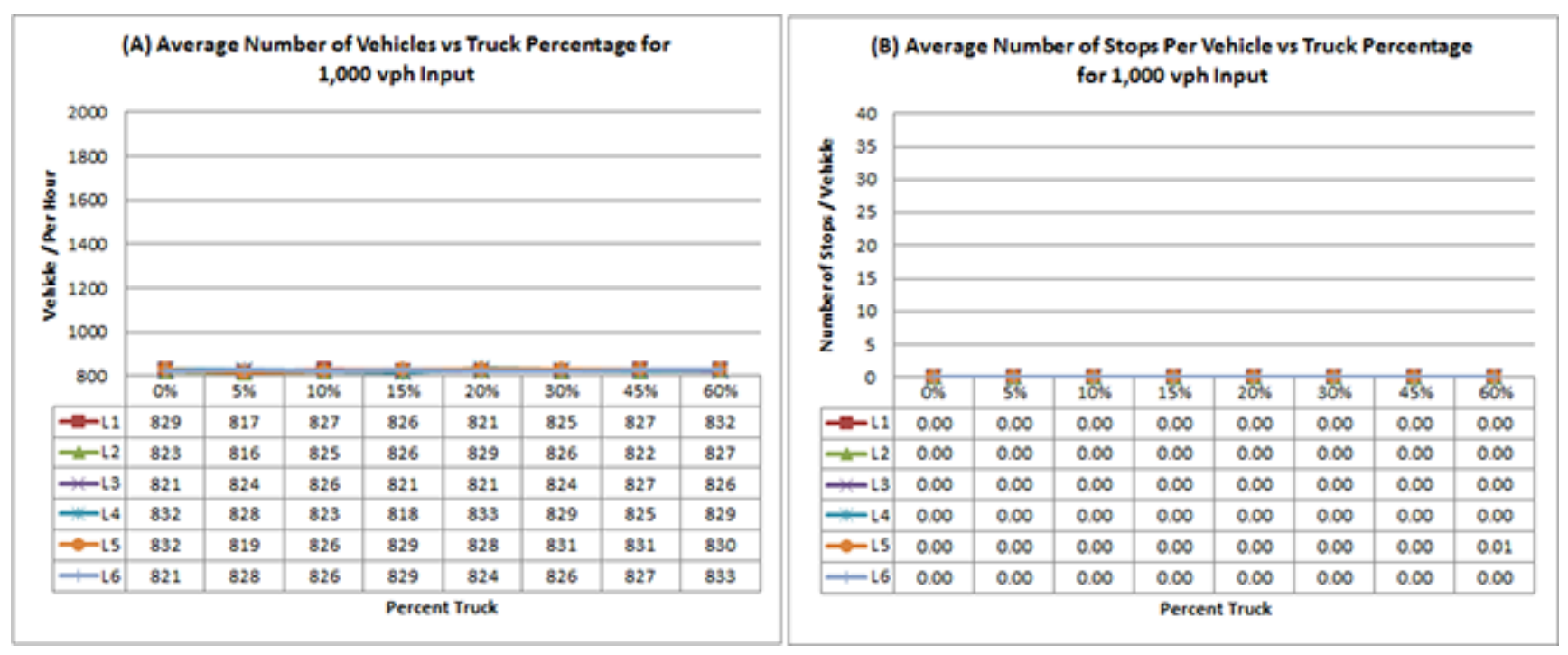

Fig. 6 Performance comparison for 1,000 vph input 

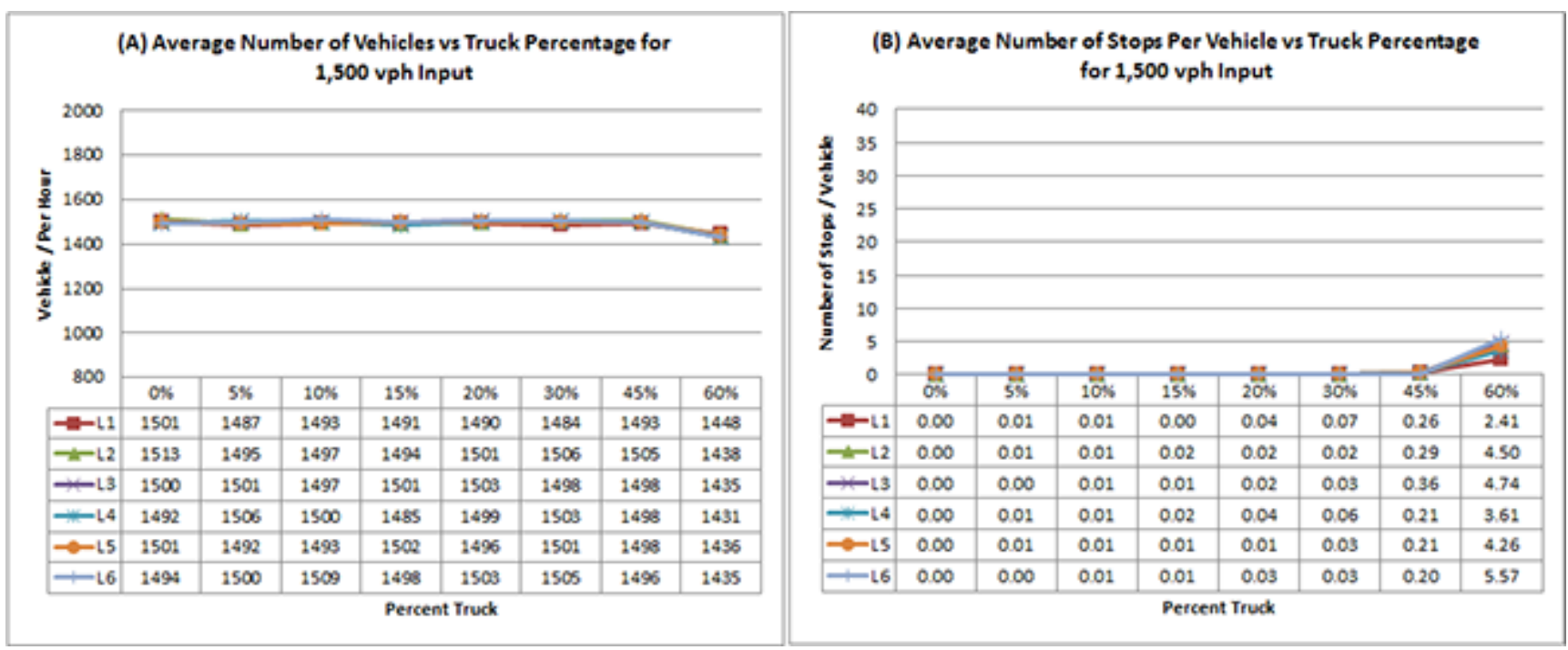

Fig. 7 Performance comparison for 1,500 vph input

truck percentages, ANOVA test for the 2,000 vph input volume revealed the taper lengths produced equal values for both throughput volume and number of stops.

\subsection{High traffic volumes (Greater than 2,500 vph)}

One notable difference is that the throughput and numberof-stop differences between truck percentages are more pronounced for the 2,000 vph and 2,500 vph input (shown in Fig. 8 and 9) when compared with the 1,500 vph input volume. For instance, the average throughput volume at zero percent truck presence at 1,500 vph input volume was 1,513 vph. For the same input volume, the average throughput volume at 60 percent truck presence was 1,448. The difference between the two is $65 \mathrm{vph}$. However, when this same procedure is performed for the higher input volume 2000 $\mathrm{vph}$, the difference between the two truck percentages is 539 vph. This coincides with the standard deviation for throughput at the 1,500 vph and 2,000 vph input levels, which were $22 \mathrm{vph}$ and $178 \mathrm{vph}$, respectively. The standard deviation for throughput volume at the 2,500 vph input level was also calculated as $176 \mathrm{vph}$. This suggests high truck presence has a variable effect on traffic flow and safety during periods of high volumes or when volumes have exceeded capacity.

\section{Conclusion}

This paper reports the outcome of 192 zipper merge simulated scenarios that consisted of six different percent truck compositions, eight different transition lengths, and four

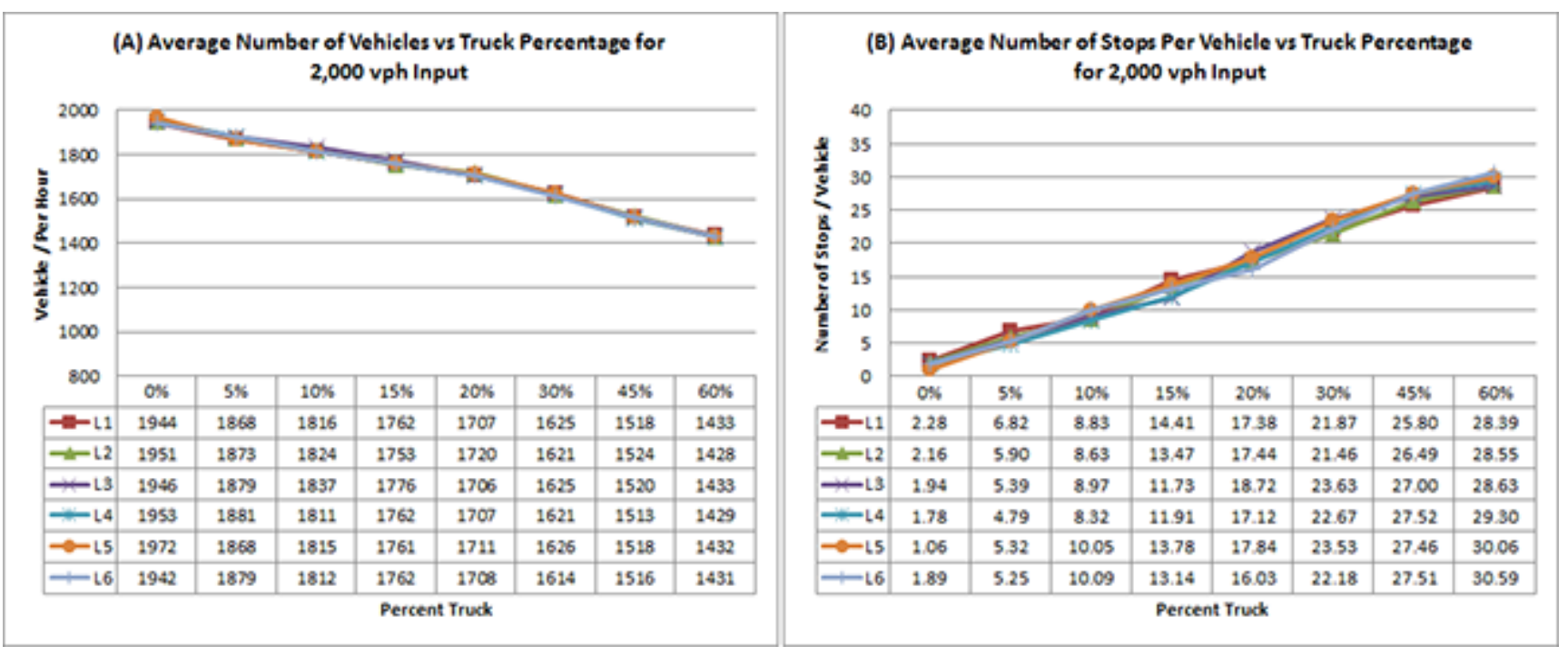

Fig. 8 Performance comparison for 2,000 vph input 


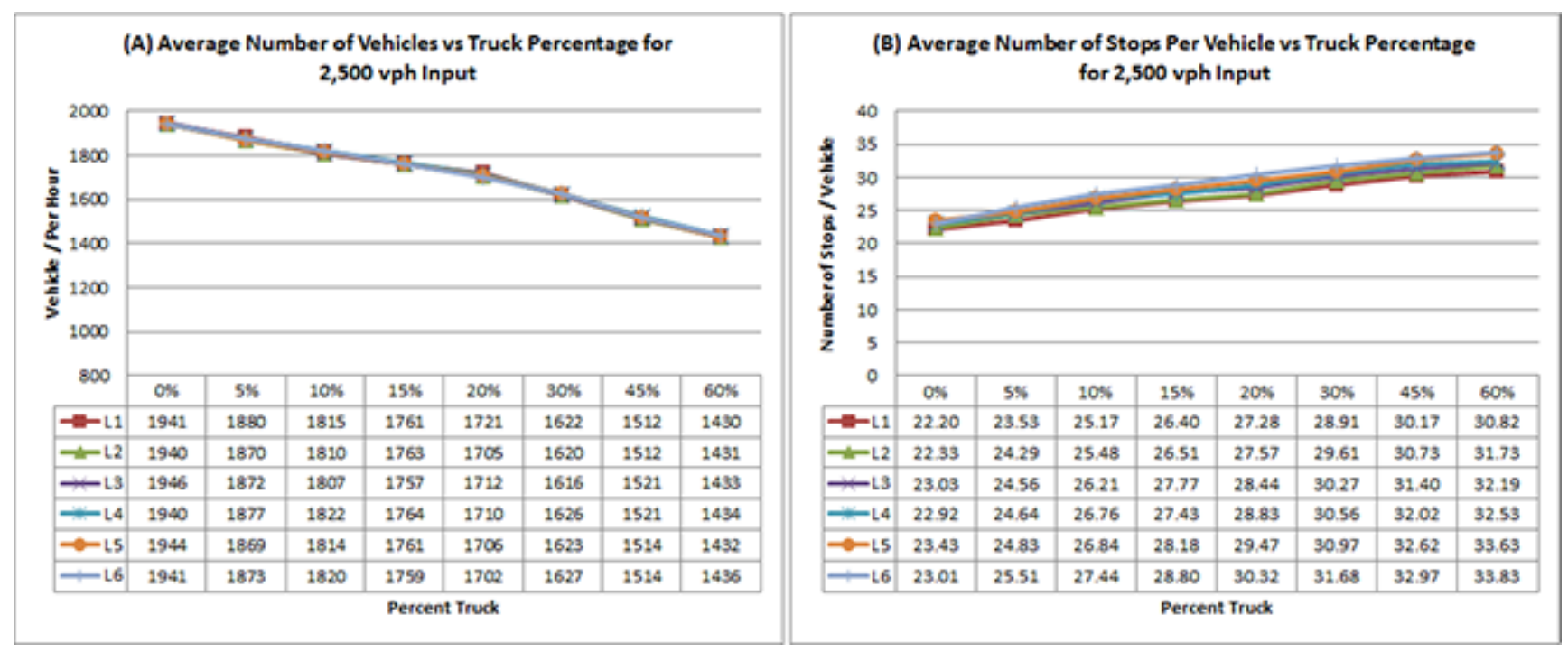

Fig. 9 Performance comparison for 2,500 vph input

different traffic volumes. The main objective of this research was to identify a taper length that was most efficient at safely moving vehicles through an area using a two-sided taper, more commonly known as the zipper merge configuration. The configuration promoted a merging environment where vehicles in each lane take turns merging. The taper length was of key concern because this unique method simultaneously merges two lanes into a center lane before shifting the lane to the right or left of construction work. While this method has been received well by motorist traveling through a zipper merge configuration, the length of taper required to safely and efficiently transition motorist has come under question. Currently, the taper length is constructed according to the MUTCD guidelines. However, some scholars believe shorter taper lengths would be more beneficial to traffic flow while others contend a longer taper length would be safer and better accommodate heavy truck traffic.

Simulation models were constructed and calibrated in VISSIM using traffic data from a live zipper merge field test. The scenarios varied by truck percentage, arriving volume, and taper length. The outputs of ten simulations with different random seeds were averaged for each scenario. Throughput volume and number of stops were the output variables used in this study to identify the optimum taper length.
Taper lengths that performed the best varied by scenario. However, there was not any statistically significant difference between the taper lengths, in terms of what their effect was on traffic. For all traffic volumes and taper lengths, truck presence reduced the operational performance of the zipper merge. Each taper length scenario with high truck percentages, resulted in lower traffic flow and increased vehicle stops. Truck percentages greater than 45 percent greatly weakened operations across all taper length suggesting 45 percent or more trucks would decrease the capacity of 2-to-1 zipper merge configurations.

The ANOVA tests performed between each taper length revealed that throughput volumes and the number of stops associated with each taper length were statistically equal. Since there were no differences between the results of each taper length, it is not likely that any of the tested lengths are a better fit over the other. This finding supports the continued use of the transition/taper length guidelines in the MUTCD for zipper merge applications because of its flexibility for a wide range of percent truck compositions, and no significant improvement of alternative options. Lengths that deviated too much from the lengths specified in the MUTCD could result in lower performing merge areas during high volume periods. 


\section{References}

Aghazadeh, F., Ikuma, L. (2013) "The Joint Merge: Improving Work Zone Traffic Flows", UTC Spotlight University Transportation Centers Program, Research and Innovative Technology Administration, U.S. Department of Transportation, [online] Available at: http://www.evaccenter.lsu.edu/pub/spot_0713.pdf [Accessed: 16 January 2014]

Bureau of Transportation Statistics (2009) "Transportation Statistics Annual Report", [online] Available at: http://www.bts.gov/publications/transportation_statistics_annual_report/2009/pdf/entire.pdf [Accessed: 02 June 2011]

California State Transportation Agency (2008) "Annual Average Daily Truck Traffic on the California State Highway System", Department of Transportation, State of California, [online] Available at: http://traffic-counts.dot.ca.gov/truck2008final.pdf [Accessed: 02 June 2011]

Chatterjee, I., Edara, P., Menneni, S., Sun, C. (2009) "Replication of Work Zone Capacities in a Simulation Model", Transportation Research Record, 2130(1), pp. 138-148. https://doi.org/10.3141/2130-17

Chitturi, M. V., Benekohal, R. F. (2008) "Calibration of VISSIM for Freeways", presented at the 87th Annual Meeting of the Transportation Research Board, Washington, D. C., USA, Jan. 13-17, 2008.

Costa, C. (2013) " Interview on Joint Merge Implementation on Highway 401 in London Ontario", Interviewed by Idewu, W. [conversation] (Personal Communication, 23 May 2013).

Dixon, K. K., Hummer, J. E., Lorscheider, A. R. (1996) "Capacity for North Carolina Freeway Work Zones", Transportation Research Record, 1529(1), pp. 27-34. https://doi.org/10.1177/0361198196152900104

Idewu, W. (2009) "Development and Operational Analysis of Highway Alternating Merge Transition Areas", PhD Thesis, Louisiana State University.

Idewu, W. I. A., Wolshon, B. (2010a) "Joint Merge and Its Impact on Merging Speeds in Lane Reduction Areas of Construction Zone", Transportation Research Record, 2169(1), pp. 31-39.

https://doi.org/10.3141/2169-04

Idewu, W., Wolshon, B. (2010b) "Effect of Vehicle Lane Position on Approaches to Construction Zone Lane-Drop Joint Merges", presented at 9oth Annual Meeting of the Transportation Research Board, Washington D.C., USA, Jan. 23, 2011.
Jiang, Y. (1999) "Traffic Capacity, Speed, and Queue-Discharge Rate of Indiana's Four-Lane Freeway Work Zones", Transportation Research Record, 1657(1), pp. 10-17. https://doi.org/10.3141/1657-02

Macaluso, N. (2008) "nterview on Merge Configurations Set Up Operations in Construction Zones", Interviewed by Idewu, W. [conversation] (Personal communication, 27 May 2008).

Maze, T. H., Schrock, S. D., Kamyab, A. (2000) "Capacity of Freeway Work Zone Lane Closures", presented at Mid-Continent Transportation Symposium, Iowa State University, Center for Transportation Research and Education, May 15-16, 2000.

McCoy, P. T., Pesti, G. (2001) "Dynamic Late Merge-Control Concept for Work Zones on Rural Interstate Highways", Transportation Research Record, 1745(1), pp. 20-26. https://doi.org/10.3141/1745-03

PTV (2009) "VISSIM 5.20 User Manual", Planung Transport Verkehr AG, Karlsruhe, Germany.

Racha, S., Chowdhury, M., Sarasua, W., Ma, Y. (2008) "Analysis of Work Zone Traffic Behavior for Planning Applications", Transportation Planning and Technology, 31(2), pp. 183-199. https://doi.org/10.1080/03081060801948175

Rayaprolu, P., Ishak, S., Qi, Y., Wolshon, B. (2013) "Operational Assessment of Joint and Conventional Lane Merge Configurations for Freeway Work Zones", Journal of Intelligent Transportation Systems, 17(4), pp. 255-267. https://doi.org/10.1080/15472450.2012.707052

Rayaprolu, P., Ishak, S., Wolshon, B. (2010) "Operational Assessment of Joint and Conventional Lane Merge Configurations for Freeway Work Zones", presented at the 89th Annual Meeting of the Transportation Research Board, Washington D.C., USA, Jan. 10-14, 2010.

Yang, N., Chang, G.-L., Kang, K.-P. (2009) "Simulation-Based Study on a Lane-Based Signal System for Merge Control at Freeway Work Zones", Journal of Transportation Engineering, 135(1), pp. 9-17. https://doi.org/10.1061/(ASCE)0733-947X(2009)135:1(9) 